> La synthèse orientée vers la diversité structurale vise à combler des « espaces chimiques » tridimensionnels laissés inoccupés par les chimiothèques traditionnelles. Grâce au développement de nouvelles stratégies de synthèse qui s'appuient sur les réactions divergentes, le chimiste est aujourd'hui capable, en seulement deux ou trois étapes de synthèse, de réaliser une telle chimiothèque qui assure l'obtention d'un nombre important de produits différents présentant qualitativement une grande diversité structurale. Nous présentons ici quelques exemples qui illustrent cette démarche vers plus de diversité et de complexité moléculaires, avec des applications concernant la découverte et l'optimisation de composés biologiquement actifs. <

De nos jours, pour découvrir ou valider une nouvelle cible biologique, une des premières démarches consiste bien souvent à se procurer une chimiothèque de petites molécules et à identifier par criblage celle(s) qui seront active(s) sur la cible ou sur la cellule entière. À ce stade, le critère qui préside souvent au choix d'une chimiothèque est « de combien de molécules dispose cette chimiothèque, en aurai-je assez pour que le criblage soit un succès? ». Ce réflexe signifie que l'on considére que l'accroissement du nombre de molécules testées influencerait de facto les chances de succès. Ce n'est pas faux, mais c'est insuffisant. II serait plus judicieux de se poser la question suivante : quelle diversité structurale caractérise cette chimiothèque? Cribler une chimiothèque qui possède la même structure centrale, c'est-à-dire dont tous les composés appartiennent à une même famille, risque de produire des réponses limitées à cette famille, alors que le but du criblage est justement d'aller chercher de nouvelles réponses. Pour combler cette lacune, il convient de disposer d'une chimiothèque qui puisse couvrir tout l'espace tridimensionnel inoccupé, tout en faisant varier les groupements fonctionnels dans toutes les directions de l'espace.

Cet article fait partie de la série «Chémobiologie » qui a débuté dans le $n^{\circ} 12$, vol. 30, décembre 2014 (www.medecinesciences.org).

\section{Chémobiologie (3) \\ Synthèse orientée \\ vers la diversité \\ structurale pour \\ explorer le vivant}

Yung-Sing Wong ${ }^{1,2}$

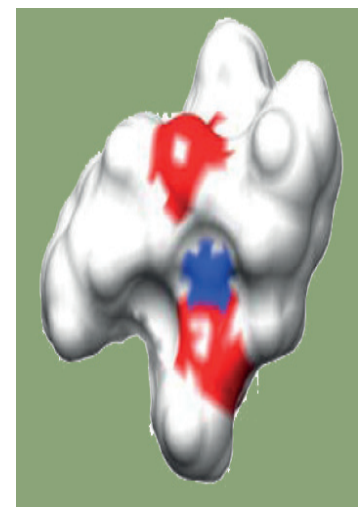

'Université Grenoble Alpes ;

${ }^{2}$ CNRS, département

de pharmacochimie

moléculaire, UMR 5063,

F-38041 Grenoble Cedex, France.

yung-sing.wong@ujf-grenoble.fr

Depuis les années 2000, des chercheurs se sont lancés ce nouveau défi, celui de la synthèse orientée vers la diversité (DOS en anglais pour diversity oriented synthesis) [1,2], qui consiste à couvrir au maximum tout l'espace tridimensionnel en élaborant des stratégies qui accroissent la diversité moléculaire tout en limitant au maximum le nombre d'étapes de synthèse. Avant d'aborder ce concept, il convient d'établir les paramètres qui définissent et quantifient la diversité structurale.

\section{Définition de la diversité structurale}

Quels sont les leviers pour créer de la diversité au niveau moléculaire? Il est possible de décomposer ces leviers en éléments de diversité pour mieux comprendre leur implication [3]. Ainsi, le chimiste s'appuie sur trois éléments principaux pour créer de la diversité structurale : la diversité liée aux blocs constitutifs, à la stéréochimie et au squelette central.

\section{Élément de diversité de fragments ou blocs constitutifs}

La synthèse du motif 1,4-dihydropyridine [4] est un bon exemple pour expliquer la diversité liée aux blocs constitutifs. Ce motif se retrouve dans bon nombre de chimiothèques de synthèse, et a déjà contribué à la découverte de nouvelles têtes de série, notamment des modulateurs de canaux calcique [5]. La condensation de $\beta$-céto-esters, d'anilines et d'aldéhydes $\alpha, \beta$-insaturés (Figure $1, \mathbf{1}, 2$ et 3 respectivement) permet d'obtenir, en présence d'un catalyseur chiral (4), des 1,4-didydropyridines (5) présentant une grande variabilité des substituants $R^{1}, R^{2}, R^{3}$ et $R^{4}$. Cette réaction est appelée réaction multicomposé (MCR en anglais pour multi-component reaction) : au moins trois réactants variables différents (ici la réaction est à trois composés $\mathbf{1 ,} 2$ et $\mathbf{3}$ ) vont réagir pour s'unir et 


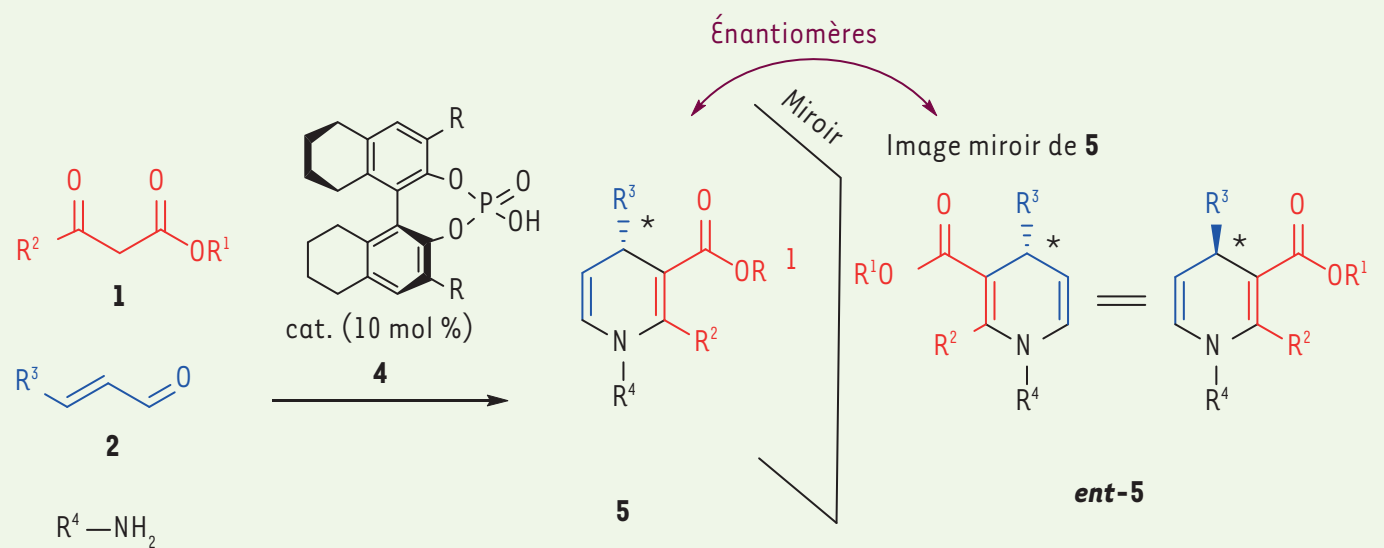

3

$73-97$ ee $\%$

Figure 1. Diversité liée aux blocs constitutifs. 1,4-didydropyridines obtenues par condensation de $\beta$-céto-esters, d'anilines et d'aldéhydes $\alpha$, $\beta$-insaturés en présence d'un catalyseur chiral.

former un produit $\mathbf{5}$ sélectivement. De la combinaison de ces réactants, une multitude de composés de type $\mathbf{5}$ peuvent être obtenus. L'inconvénient de cette approche est que la diversité moléculaire est restreinte à la variation des blocs constitutifs autour d'un squelette central commun. Les réactions de ce type «multicomposés » ont été très largement employées pour créer de grandes chimiothèques, qui présentent cependant peu de variabilité tridimensionnelle.

\section{Élément de diversité stéréochimique}

Dans l'exemple précédent, il faut remarquer que le produit final possède un nouveau centre asymétrique (symbolisé par *) et que la réaction a été réalisée dans des conditions énantiosélectives, c'est-à-dire que seul l'énantiomère $\mathbf{5}$ est obtenu (I'autre énantiomère ent-5, qui est son image en miroir, n'est, lui, pas formé). Les énantiomères ${ }^{1} \mathbf{5}$ et $\mathbf{e n t} \mathbf{- 5}$ sont deux molécules structurellement différentes car non superposables. Le fait de créer des centres asymétriques (appelés aussi centres sp3) génère de la complexité moléculaire, et donc accroît facilement la diversité moléculaire. Sans le centre asymétrique *, le produit $\mathbf{5}$ serait plan (dans le plan de la feuille). Grâce à ce centre, le groupement $\mathrm{R}^{3}$ sort du plan (en arrière du plan de la feuille) et la molécule prend ainsi une forme plus «tridimensionnelle ». La chiralité ${ }^{1}$ est donc un facteur d'accroissement de la complexité tridimensionnelle qui accentue le potentiel de diversification morphologique. Des chercheurs ont récemment démontré qu'accroître le nombre de centres chiraux dans une chimiothèque avait un impact positif sur la sélectivité et la fréquence des hits [6].

Au cours d'une réaction, le chimiste est capable de créer plusieurs nouveaux centres asymétriques en ayant un contrôle sur chacun d'eux. Dans le contexte de la diversification spatiale, le défi est de réaliser de la divergence stéréochimique, c'est-à-dire d'aboutir à un squelette central identique, mais qui présente des stéréochimies (les centres chiraux) différentes

\footnotetext{
${ }^{1}$ Chiralité : du grec $\chi \varepsilon l \rho$ (la main). Un objet chiral n'est pas superposable à son image dans un miroir plan. Un exemple est celui des deux mains. L'objet chiral et son image sont des énantiomères. Deux stéréoisomères qui ne sont pas des énantiomères sont des diastéréo-isomères.
}

(les produits obtenus sont alors des diastéréoisomères). Leur substituants sont donc orientés dans différentes directions de l'espace, ce qui aboutit à des formes tridimensionnelles nouvelles. La réaction multicomposé de Petasis illustre parfaitement cette notion de divergence stéréochimique qui est réalisée de façon simple, sélective et efficace (Figure 2) [7]. Le dérivé d'acide aminé 6, possédant un centre chiral (*) et le réactant acide boronique 7 sont mis en réaction avec le dérivé chiral 8 ou son énantiomère ent8, ce qui conduit à former sélectivement et respectivement $\mathbf{9}$ ou 10. Les composés 9 et $\mathbf{1 0}$ sont diastéréoisomères l'un par rapport à l'autre et ont une morphologie tridimensionnelle différente, comme l'illustrent les représentations à droite de la Figure 2. Leur nouveau centre asymétrique (*) est totalement contrôlé par l'induction chirale du centre asymétrique portée par 8 ou ent-8.

\section{Éléments de diversité de squelette}

Le chimiste peut aussi explorer la diversité morphologique à travers la formation de différents squelettes centraux. Un très joli exemple est illustré par le précurseur central 11 (Figure 3) [8]. En seulement une étape à partir de $\mathbf{1 1}$, et selon le réactif utilisé, voire dans certains cas sa quantité, il est possible d'aboutir à une grande variété de squelettes qui présentent des morphologies linéaires, circulaires ou globulaires.

\section{Recombinaison des éléments de diversité : la stratégie multi-étapes assemblage /couplage/ appariement (build/couple/pair)}

Bien souvent, une chimiothèque est conçue en plusieurs étapes de synthèse. Pour atteindre une morphologie variée en peu d'étapes, la synthèse orientée vers la diversité 


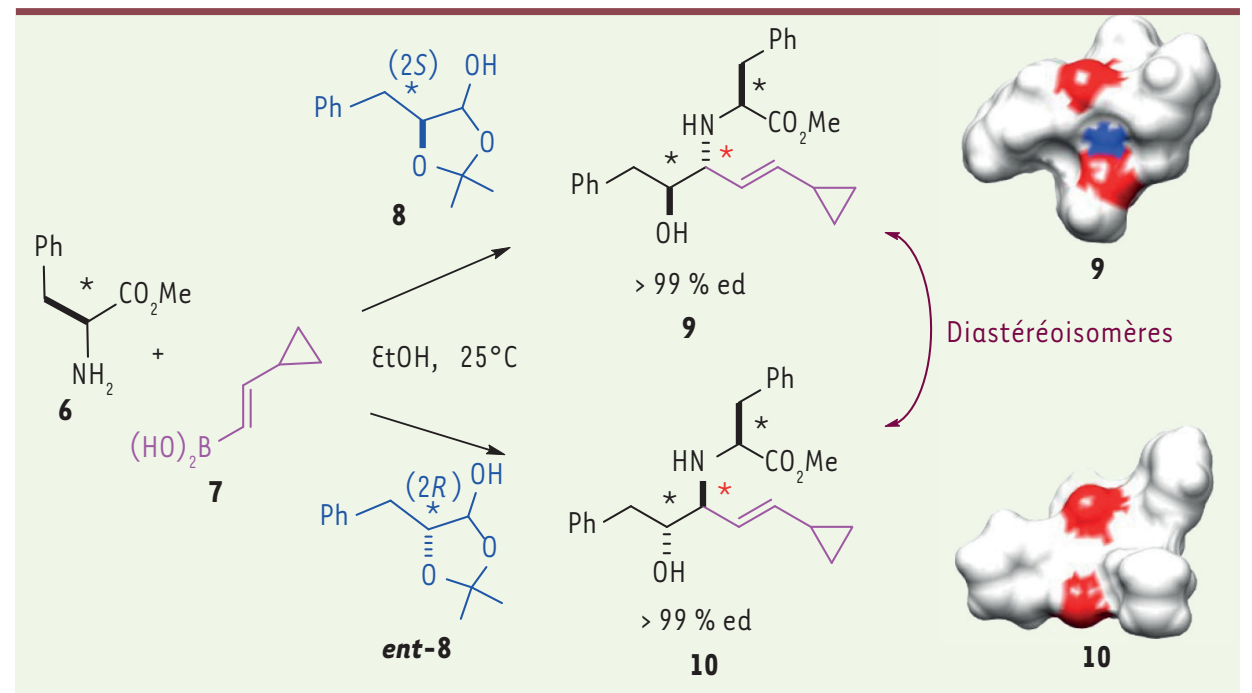

Figure 2. Réaction multicomposé de Petasis et notion de divergence stéréochimique.

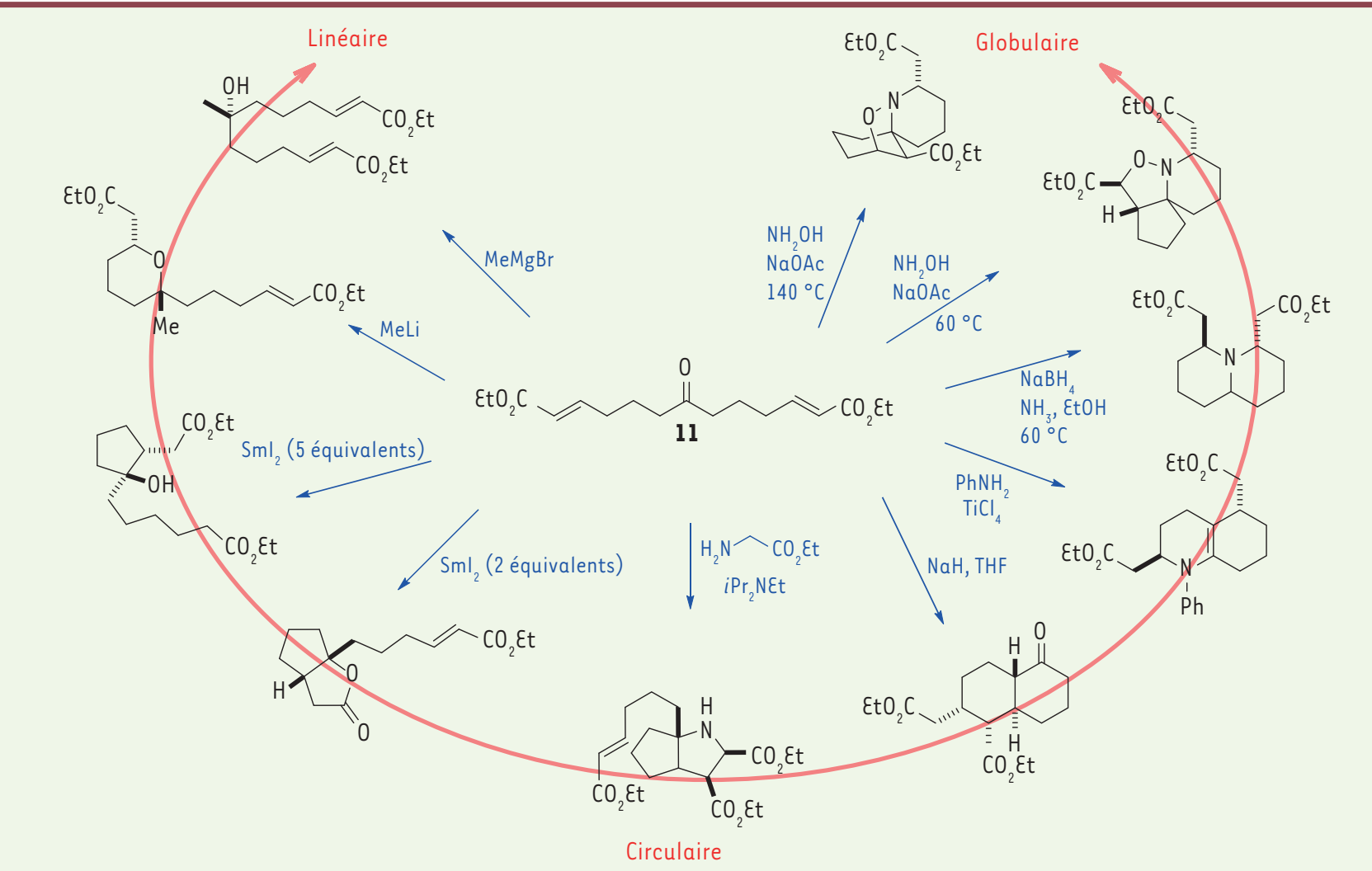

Figure 3. ćléments de diversité de squelette. Créer une grande variété de formes tridimensionnelles en une ou deux étapes à partir d'un précurseur central 11.

exploite la possibilité de combiner les éléments de diversité entre eux. Une des approches appelées BCP (build/couple/pair) consiste à assembler d'abord les fragments variables (assemblage, par exemple ici la réaction multicomposé de Petasis, Figure 4) pour créer un motif central, puis à greffer sur ce dernier un nouveau fragment variable (couplage, soit par le fragment 12 ou 13) possédant des fonctionnalités réactives latentes différentes, et capable de réagir ultérieurement avec d'autres fonctions de la molécule (appariement) pour aboutir à de nouveaux squelettes inédits [9].
Les stratégies de divergence, qu'elles soient stéréochimique (la diastéréodivergence de la réaction multicomposé de Petasis à la $1^{\text {re }}$ étape) ou de squelette (ze étape et plus), induisent à chaque fois un profond remodelage tridimensionnel sur les molécules obtenues.

Il est possible d'évaluer la morphologie de chaque molécule en calculant son moment d'inertie principal, qui, une fois normalisé, peut être représenté sur un graphe 


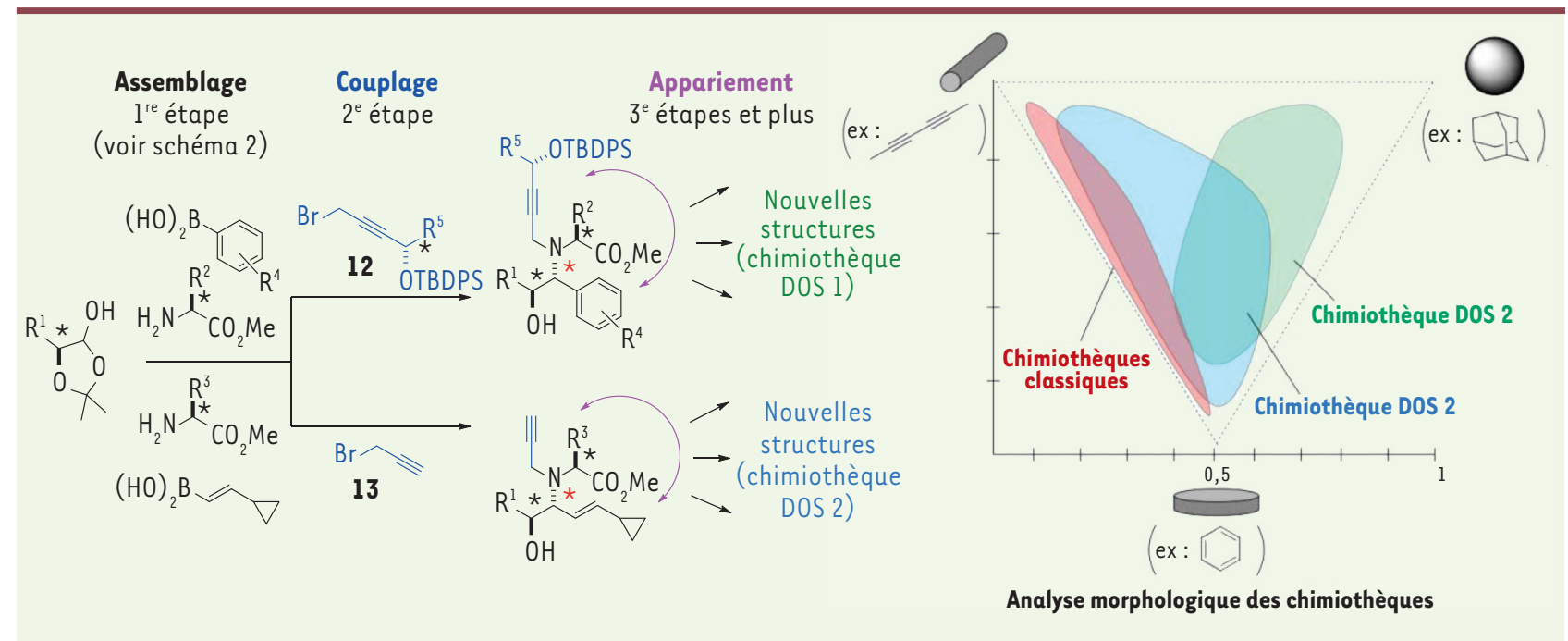

Figure 4. Recombinaison des éléments de diversité. Évaluation de la morphologie de chaque molécule représentée sur un graphe par le calcul de leur moment d'inertie principal.

sous la forme d'un triangle (Figure 4) [10,11]. Ainsi, les molécules situées dans la partie en haut à gauche ont une forme plutôt linéaire, celles en bas plutôt plane, et celles en haut à droite, globulaire. Les chimiothèques de synthèse dites classiques (en rouge) sont localisées la plupart d'entre elles dans la zone comprise entre la forme linéaire et plane.[10] Dans le cas de notre exemple, deux chimiothèques différentes ont été produites en 3 à 5 étapes seulement. La chimiothèque DOS 2 (en bleu) couvre une bonne partie gauche du triangle, alors que la chimiothèque DOS I (en vert) occupe l'autre moitié (plus globulaire). Ainsi, à partir de précurseurs communs, il est possible d'occuper quasiment tout l'espace tridimensionnel en peu d'étapes synthétiques pour aboutir à une chimiothèque morphologiquement diverse.

\section{Applications à la découverte de nouveaux modulateurs épigénétiques : les inhibiteurs d'histone désacétylases}

L'approche BCP a été utilisée pour créer une chimiothèque de plus de 14400 macrocycles présentant une grande diversité au niveau de leur stéréochimie et de leur squelette central [12]. Leur criblage a permis la découverte d'une nouvelle classe d'inhibiteurs d'histone désacétylase (HDAC). Cette chimiothèque occupe un espace chimique qui peut être rapproché de celui de la chimiothèque DOS 1 (en vert) (voir exemple précédent, Figure 4), c'est-à-dire que les molécules présentent un aspect qui oscille entre les formes plan et globulaire.

Dans un autre registre, il est possible d'appliquer les principes de la synthèse orientée vers la diversité, en termes d'économie d'étape et de diversification, à une approche orientée vers une structure plus ciblée (target oriented synthesis [TOS]) ou pour des applications plus médicales (function oriented synthesis [FOS]) [13].

Notre laboratoire s'intéresse à l'analyse et à la compréhension des relations structures-activités des inhibiteurs d'HDAC appliqués au traitement de maladies parasitaires intracellulaires, en particulier celles qui sont liées à la famille des Apicomplexes (notamment Toxoplasma gondii et Plasmodium falciparum, agents responsables respectivement de la toxoplasmose et du paludisme). Le but est d'optimiser un composé qui puisse agir sur le parasite sans toucher I'hôte. Dans cette optique, nous nous sommes inspirés d'un produit naturel cyclotétrapeptide, qui présente une très bonne activité inhibitrice sur T. gondii. L'index thérapeutique reste néanmoins faible. C'est dans ce contexte que nous avons développé une stratégie, inspirée de la synthèse orientée vers la diversité, qui nous permette à la fois ( 1 ) de combiner les fragments variables à volonté entre la partie cyclopeptidique (Figure 5, 14, en gris) avec soit une partie cétonique (15, en bleu), soit une partie thioester (16, en violet), en 1 à 2 étapes, pour donner respectivement 17 ou 18 ; et (2) de modifier leur squelette cyclotétrapeptidique à 12 chaînons en depsipeptide cyclique à 15 chaînons (19) en seulement une étape [14].

À partir de cette chimiothèque réduite, nous avons pu sonder efficacement notre cible (HDAC3) et obtenir de nouvelles données sur les relations structures-activités. Parmi les nouveaux inhibiteurs $\mathbf{1 7}$, certains avaient une activité inhibitrice nanomolaire sur des cellules et un meilleur index thérapeutique que celui du produit naturel de réference. De façon inattendue, certains des dérivés thioester $\mathbf{1 8}$ ont montré une sélectivité inversée, c'est-à-dire une inhibition forte sur les cellules humaines sans effet sur le parasite. $\mathrm{Si}$ I'on modifiait le squelette des composés bioactifs les plus représentatifs $\mathbf{1 7}$ et $\mathbf{1 8}$ en dérivés cyclodepsipeptides $\mathbf{1 9}$, ceux-ci avaient perdu leur activité inhibitrice, soulignant le rôle primordial de la structure cyclotétrapeptide dans l'efficacité de ces molécules in cellulo. 


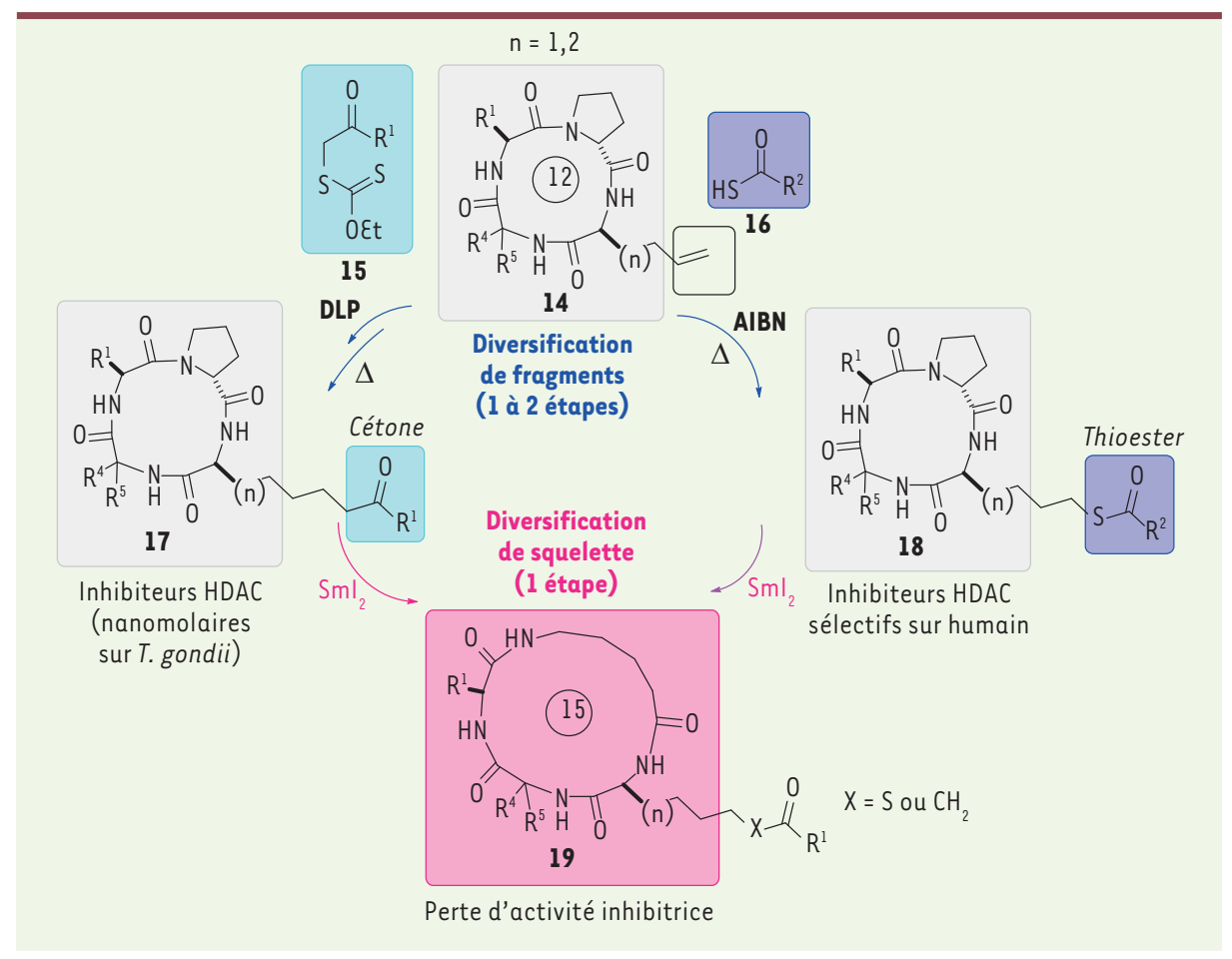

Figure 5. Stratégie inspirée de la synthèse orientée vers la diversité en vue de faciliter la pharmacomodulation d'inhibiteurs d'histone désacétylase.

\section{Conclusion}

Les performances des plate-formes robotisées actuelles offrent des perspectives de criblage à très haut débit. Pour que les campagnes de criblages soient encore plus performantes, il est opportun d'être très attentif à la qualité de la chimiothèque (grandes diversités structurales et fonctionnelles). Grâce à des outils d'analyse de la diversité moléculaire, il nous est possible d'évaluer cette qualité. Celle-ci est garante d'une chimiothèque riche en contenus d'informations structures-activités concernant la cible biologique visée. La synthèse orientée vers la diversité a pour but de concevoir des stratégies de synthèses nouvelles, qui permettent d'accéder facilement à des chimiothèques réellement diversifiées et riches en informations pour accélérer la découverte d'entités bioactives innovantes. $\diamond$

\section{SUMMARY}

Structural diversity oriented synthesis to explore the living world

Structural diversity oriented synthesis aims to fulfill the unoccupied tridimensional "chemical space" gap left by traditional chemical libraries. Through the development of novel synthetic strategies relying on divergent reactions, chemist is now able to realize in only two or three steps such library that ensures the access of a large number of products having a good quality in term of structural diversity. A few examples are presented to illustrate how this can be done in the context of increasing molecular complexity and diversity devoted to the discovery and optimization of bioactive compounds. $\diamond$

\section{LIENS D'INTÉRÊT}

Les auteurs déclarent n'avoir aucun lien d'intérêt concernant les données publiées dans cet article.

\section{RÉFÉRENCES}

1. Schreiber SL. Target-oriented and diversity-oriented organic synthesis in drug discovery. Science $2000 ; 287$ : 1964-9.

2. Burke MD, Schreiber SL. A planning strategy for diversity-oriented synthesis. Angew Chem Int Ed $2004 ; 43$ : 46-58.

3. Peuchmaur $M$, Wong YS. Expanding the chemical space in practice: diversityoriented synthesis. Comb Chem High Throughput Screen 2008 ; 11 : 587-601.

4. Wan J-P, Liu Y. Recent advances in new multicomponent synthesis of structurally diversified 1,4-dihydropyridines. RSC Adv 2012; 2 : 9763-77.

5. Edraki N, Mehdipour AR, Khoshneviszadeh M, Miri R. Dihydropyridines: evaluation of their current and future pharmacological applications. Drug Discov Today 2009 ; 14 : 1058-66.

6. Clemons PA, Bodycombe NE, Carrinski HA, et al. Small molecules of different origins have distinct distributions of structural complexity that correlate with protein-binding profiles. Proc Natl Acad Sci USA 2010 ; 107 : 18787-92.

7. Kumagai N, Muncipinto G, Schreiber SL. Short synthesis of skeletally and stereochemically diverse small molecules by coupling Petasis condensation reactions to cyclization reactions. Angew Chem Int Ed 2006; 45 : 3635-8.

8. Robbins D, Newton AF, Gignoux C, et al. Synthesis of natural-product-like scaffolds in unprecedented efficiency via a 12 -fold branching pathway. Chem Sci $2011 ; 2: 2232-5$.

9. Muncipinto G, Kaya T, Wilson JA, et al. Expanding stereochemical and skeletal diversity using Petasis reactions and 1,3-dipolar cycloadditions. Org Lett $2010 ; 12: 5230-3$.

10. Sauer WHB, Schwarz MK. Molecular shape diversity of combinatorial libraries: a prerequisite for broad bioactivity. J Chem Inf Comput Sci 2003 ; 43 : 987-1003.

11. Kopp F, Stratton CF, Akella LB, Tan DS. A diversity-oriented synthesis approach to macrocycles via oxidative ring expansion. Nat Chem Biol $2012 ; 8: 358-65$.

12. Marcaurelle LA, Comer $\varepsilon$, Dandapani $S$, et al. An aldol-based build/couple/ pair strategy for the synthesis of medium- and large-sized rings: discovery of macrocyclic histone deacetylase inhibitors. J Am Chem Soc 2010; 132 : 16962-16976.

13. Wender PA, Verma VA, Paxton TJ, Pillow TH. Function-oriented synthesis, step economy, and drug design. Acc Chem Res 2008 ; 41 : 40-49.

14. Traore M, Mietton F, Maubon D, et al. Flexible synthesis and evaluation of diverse anti-apicomplexa cyclic peptides. J Org Chem 2013; 78 : 3655-75.

\section{TIRÉS À PART}

Y.S. Wong 